\title{
Combining Micro Technologies and Unmanned Systems to Support Public Safety and Homeland Security
}

\author{
Richard E. Baker \\ Center for Unmanned Systems and Human Capital Development, Indiana State University, Indiana 47809, USA
}

\begin{abstract}
Previously, the military establishment has been the primary developer and user of micro technologies associated with unmanned systems. As these technologies become available commercially, a need exists to integrate the use of the technology into local or regional public safety and homeland security incidents. The purpose of this presentation is to explain several key factors to consider when using micro technologies and unmanned systems in support of public safety and homeland security officials. Real time information is critical to the decision making process for public safety and homeland security officials to make assessments and quickly resolve crisis situations. Unmanned micro-vehicles and micro technologies are well suited to remotely observe, gather essential information, and immediately relay it to incident responders. These technologies can provide extremely important support during responses to hostage situations, hazardous environments, search and rescue, natural disasters, border patrol and many others. The true benefit is having remote resources providing real time support to incident responders. This paper discusses the use of several different types of micro-vehicle platforms in public safety scenarios and their use of associated technologies such as GPS (Global Positioning System) autopilot, communication, and sensor devices.
\end{abstract}

Key words: Public safety, first responders, unmanned systems, improved incident response.

\section{Introduction}

Currently, unmanned vehicle systems are in limited use for public sector missions worldwide. The development of civilian unmanned technology in the United States currently lags behind military unmanned technology development largely because of unresolved regulatory and technological issues [1]. Traditionally, it has been the military establishment which has led the way in emerging unmanned technologies. While the military remains on the forefront and continues to be one of the biggest users of unmanned technologies, non-military applications, especially those of small and micro vehicles, are gaining in popularity and usage [2]. These unmanned vehicle systems have the potential to augment civil disaster and emergency incident response and recovery efforts. Optimal design of land and aerial systems for such applications will lead to

Corresponding author: Richard E. Baker, $\mathrm{PhD}$, assistant professor, research fields: aviation human factors, human factors in unmanned systems and commercial applications of unmanned systems. E-mail: richard.baker@indstate.edu. unmanned vehicles which provide the greatest potential for relief and response to emergency and disaster events while addressing public safety concerns and regulatory requirements [1].

Progressive disaster and emergency response services in the United States are supported by modern networks of law enforcement, fire protection, military, the Department of Homeland Security, and other special service personnel. UGV (unmanned ground vehicle) systems have actually been used for support work in hazardous situations for over a decade [3]. The concept of implementing UAV (unmanned aerial vehicle) systems to assist their efforts is not a new idea, but, has been proposed for many years [4]. To effectively utilize these systems requires best possible design of several different types of micro-vehicle with associated technologies such as GPS (global positioning system), autopilots, communications, and sensor devices. 


\section{Applications for Public Safety and Homeland Security}

Small and micro technologies have immediate applications for public safety and homeland security [2]. Police forces in particular from around the world are becoming increasingly interested in using small unmanned systems in their activities. They may be used to track, survey, or simply monitor activities in a geographical area. In many cases, they are able to move or interact with objects on the ground [5].

UGVs that support activities for public safety and homeland security are usually small and lightweight for mobility. They can be easily adapted to the latest security requirements according to the mission. They employ highly modular design and can be rapidly fitted with various sub-assemblies [5]. Most UGVs are controlled through a remote operator's transmitter and an onboard receiver. Images and data are returned from the vehicle via an onboard transmitter to the operator's receiver [2]. GPS information, if included, is a key factor in data location and analysis. Typical uses include explosive disposal of suspicious packages or device processing, reconnaissance and surveillance of sensitive sites, sampling and collection of hazardous materials such as chemical, biological, radiological, and nuclear materials [5].

Small UAVs are usually less than 55 pounds and are able to carry one or more payloads such as cameras or sensors. A MAV (micro air vehicle) may be as small as a few centimeters in length and wingspan. Small and micro UAV systems may be controlled manually or autonomous depending upon design characteristics and mission requirements. UAV systems designated small or micro by the manufacturer sometimes overlap in size because there is no single authoritative definition of sizes. They have different levels of automation which can be utilized to control the vehicle, and, each system needs a minimum of two processors, one for a remote transmitter and one for an onboard receiver [2]. To provide value as remote eyes and ears for the operator, each UAV must have the ability to transmit images and/or data as well as receive. The operator must be able to receive the transmission and view it for improved situational awareness and store it for further analysis [6]. GPS information provides improved data analysis and is critical for vehicles in an autonomous navigation mode using an autopilot. Although small, these technologies can have large impact. They can provide immediate and time critical information to public safety individuals during disaster or emergency crises, and, may be applied to other services such as highway patrol, traffic routing, and utilities monitoring [1].

\section{The Potential for Improved Information for First Responders}

Disaster and emergency response and relief efforts include all actions by first responders and subsequent assistance efforts during and immediately following a catastrophic event. The primary purpose of response and relief efforts is to save human lives. Secondary purposes are to preserve and maintain the environment, protect property, keep the peace, and uphold governmental authority. In any event, incident commanders and crisis leaders have a critical need for information to support their situation awareness and to formulate effective response efforts [6].

Contemporary manned response and recovery systems include ground vehicles, conventional fixed-wing aircraft, and rotary-wing aircraft. However, UAV systems have the greatest potential to improve the effectiveness and capabilities of first responders by enhancing and providing advanced predictive capabilities and early warning [7]. These new UAVs may be carried by an individual, launched by hand, and may include GPS navigation with an autopilot. A wide variety of system types of all sizes with varying capabilities already exist with even more under development [1].

Public safety or homeland security events for which UAV systems could be implemented include, but are not limited to: severe storms, tornados, hurricanes, 
wild fires, tsunamis, floods, earthquakes, avalanches, civil disturbances, oil or chemical spills, and urban disasters [8]. Advanced vision systems can provide ground operators a clearer picture of developing scenarios and offer a greater breadth of situational awareness for the incident commander resulting in more efficient and effective responses [1].

The DHS (Department of Homeland Security) is already using Predator-Bs by General Atomics Aeronautical Systems, San Diego, C.A., to patrol U.S. borders with Mexico and Canada, review flood waters along the Mississippi River [9], and to obtain IR (infrared) pictures of major wild fires [10].

While Predators are larger aircraft, small UAVs and even micro-systems are being tested and incorporated into the missions of USCBP (U.S. customs and border protection) and the U.S. Coast Guard. With UAV technology, the USCBP can have someone watching a monitor at headquarters and agents in the field can be deployed to an area where there is likelihood of encountering illegal border crossers. USCBP calls the UAV technologies force multipliers [11]. The new small and micro-technology UAVs are small enough to be carried by individual personnel in the field and hand launched.

One approach already underway is the research and development of MAV (micro-aerial vehicles) at Wright State University under the guidance of Dr. George Huang. In a personal interview on June 6, 2011, Dr. Huang stated the goal of his team is to develop and build micro-aerial vehicles small enough to enter a building, go into each room, and then conduct surveillance operations. According to Dr. Huang, the size is very important to enter small spaces such as doing search and rescue operations under building rubble after an earthquake. With a micro aerial vehicle, just the size of an insect, first responders can send it in, make contact with the people, even take pictures or audio of the situation inside, and help the responders understand what needs done. MAVs can help us locate, excavate, and save survivors.
To develop the MAV, Dr. George Huang and his team studied the dragonfly and the ladybug. From each, they determined how the insects sense air flow direction and ascend or descend as needed to conserve energy. While the MAV is currently radio controlled, Dr. Huang hopes to develop sensors for the body of the MAV so it can determine flow direction and react autonomously. The team's goal of miniaturization also presents major challenges in small power and sensor payloads.

\section{Preparing Operators and Analysts as Part of the System}

With regard to public safety, homeland security and the use of micro-technology systems, training the system operators must come first. Several means are available to accomplish effective training. On-site or manufacturer training classes can be arranged. Some university programs may offer short courses on UAS operations. Military training is probably considered to be the best [2].

Analysts training may be more difficult to accomplish. Given the highly specialized nature of photo/sensor analysis interpretation, limited avenues are available to receive such training. Many analysts receive training from military or government programs. As stated by Mr. Hugh Bolton, President and CEO of the Advanced Technology Intelligence Center, Dayton, $\mathrm{OH}$, in a personal interview on June 6, 2011, some private sector training centers exist, but these centers may require security clearances in order to receive the training.

The technologies required to present the obtained data to the analysts and the decision makers include the vehicles, an expanded and enhanced communications network, and dynamic databases working cohesively as a living network. This network serves to relay data to the analysts and decision makers as well as interface with first responders on site [1]. In addition, these technologies include small and micro aerial, ground, and marine vehicles as carriers of the initial sensors. 
Many first responder agencies actively conduct practice exercises with the technologies to become familiar and more efficient in their operations.

As has been emphasized, timely and accurate information is the key factor in the decision making process. Public safety and homeland security leaders must be able to have speedy access to this information to effectively direct the response to a crisis situation [8]. With effective training, the small and micro-technology systems can provide decision makers with essential remote eyes and ears for the critical information link in the process.

\section{Key Factors to Consider}

The benefits of using small and micro-systems are many. Most are extremely portable and can be deployed in a relatively short period of time. This allows an operator to move a system, whether ground or aerial, directly to an area of concern and launch the vehicle immediately. Bigger systems might take longer to prepare and launch, hence, they take more time to gather critical information. Information about an event or situation is time sensitive and essential to any decision maker. Smaller systems may be able to operate lower to the ground and move in and out of confined areas of operation better than larger systems. Just as in larger systems, small UAV systems are reprogrammable and can operate autonomously utilizing autopilots, GPS, and earth mapping. The sensors can include high definition filming with EO (electro-optical) and IR (infrared). Other information gathering devices may be utilized as dictated by the mission specific requirements.

Unfortunately, very small technologies are often misunderstood. With a great deal of hype about micro-technology, some enthusiasts say it will solve the all the world's problems. In contrast, critics cite concerns ranging from regulatory and intellectual property issues to the idea of self-aware, self-replicating machines that would ultimately take over the world [12].
While some police forces have been interested in exploring the possible use of UAVs for law enforcement and public safety, the FAA (Federal Aviation Administration) remains very reluctant to allow their widespread use. It has, in at least one case, shut down the testing of UAVs by a local police department [13]. As stated previously in this paper, there are numerous applications for micro-systems in support of public safety and homeland security. At this juncture, several obstacles exist to allow easy implementation of the UAVs.

First, the methods to properly train, educate and deploy the micro-systems on a regular basis with proficiency and precision are still lacking. Operators must continually hone their skills lest they become non-proficient and lack confidence in skill sets. Of the major issues preventing first responders from using unmanned systems, two of the largest are SA (situational awareness) and user interaction [3]. Training, practice, and skill building can address these two issues.

Another obstacle is cost and expense. The costs of operating large unmanned systems can become more than double the costs of operating a manned system. Operating one UAV can take a crew of several Border Patrol agents for a Predator system. In contrast, the small and micro UAVs require a much smaller cost in terms of personnel support with the higher costs in miniaturization [11].

Acquiring the resources and financing to purchase these complex and expensive systems presents a major problem for most organizations. Simply making a system smaller does not necessarily make that system any less expensive. Many of the smaller systems can start at \$50,000.00 and even higher. The sensors and cameras are often in the tens of thousands of dollars, too, and may even exceed the cost of the vehicle in some cases. The lack of funding opportunities and reduced budgets certainly can make these systems difficult to acquire and maintain. 
In his interview, Dr. Huang also stated that, generally speaking, there is a definite price to pay for miniaturizing the system. As the system or vehicle platform become smaller, the payload must be lighter and mission time becomes shorter. Additionally, when using small systems over full sized systems, weather conditions become a big factor in mission success or completion. The smaller systems are much more susceptible to wind, temperature and relative humidity. A small or micro UAV uses much of its power to fight wind strong currents or may be completely swept away. Larger systems with bigger and stronger propulsion capabilities can overcome the weather, carry larger payload, as well as possibly have increased loiter time.

Another limitation for both large and small unmanned systems is dealing with the FAA and airspace restrictions. While efforts are underway to integrate unmanned systems into the NAS (national airspace system), the inability of the vehicles to see and avoid other aircraft is a major stumbling block. Hence, the FAA has placed very strong restriction as to the altitudes and areas of operation on unmanned vehicle operations.

As with other similar systems, small and micro technology unmanned systems are most often very complex. They can become more complex by the engineering of smaller, lighter and stronger components. Given the smaller size of the components, they are inherently more difficult to repair. Special diagnostics are often required in order to pinpoint faulty components. In light of the complexities, repair may require the entire system or vehicle be returned to the manufacturer. Depending on the situation, the result may be quite expensive and might create down time or loss of capability for an extended period of time.

Interfacing can provide special challenges as well. Many parts of a small or micro system rely on inputs and information from different subsystems. For example, what may first seem to be a simple problem with a flight control might be rooted in the control itself, an actuator, a receiver or even a radio controller. If it is in fact the radio controller, then various other subsystems will be affected as well. Interference from outside sources must also be anticipated and managed. Depending on the system, electrical and microwave sources may play havoc with respect to control inputs or even live streaming video from the vehicle. Operators are always on the alert for possible interference.

\section{Micro Technologies Integration at Indiana State University}

ISU (Indiana State University) Aviation has recently created an Unmanned Systems program to meet the ever increasing demand for training and human capital development in the field of unmanned systems. The focus at ISU is not just on the UAS (unmanned aerial systems), but on all unmanned systems to include ground and marine systems as well. Students at ISU are exposed to the individual systems as well as are introduced to the integration of the different systems and how they complement each other during real world scenarios.

ISU utilizes specialized teaching methods to cover micro systems and sensors associated with rotary, fixed wing, lighter than air, tracked vehicles and marine systems. The intent is to demonstrate how all systems can work in sync and provide timely information which is critical to decision-makers. One scenario currently under development is to have a 17 foot airship execute an airdrop of a micro tracked unmanned ground vehicle into a potentially hostile or contaminated area and, in turn, and have both vehicles transmit live streaming video of the developing situation from multiple perspectives. While certain limitations must be overcome, students are exposed to the concept of small and micro technologies, multiple sensor systems, and their integration into scenario based training situations.

In addition to the student training and education at ISU, the university has also just created a new Center for Homeland Security and Crisis Leadership. This 
center works closely with the Aviation Technology Department to integrate unmanned systems micro technologies into scenarios used to train crisis leaders. As noted earlier, these resources are critical with respect to providing real time information for officials in public safety and homeland security. Proper utilization of these components can help to make quick assessments and resolve crisis situations.

\section{Conclusions}

In the preceding pages, identified were many applications of micro-technologies in unmanned systems and how this technology may support crisis leaders associated with public safety and homeland security. As an emerging technology, small and micro unmanned systems can be useful in building situational awareness, overall assessment and delivering critical information. While there are certain complexities and challenges with these types of systems, the advantages of using them far outweigh any obstacles which may be encountered. Integrating small and micro technology unmanned systems into any situation as an information source takes proficiency and coordination among the participants. Without this, the advantages of these systems can quickly disappear. As small and micro unmanned systems become more readily available and are more often used in the public safety and homeland security sectors of our society, confidence levels will rise and demand for such technologies will increase.

\section{References}

[1] W. M. DeBusk, Unmanned aerial vehicle systems for disaster relief: Tornado alley, available online at: http://ntrs.nasa.gov/archive/nasa/casi.ntrs.nasa.gov/20090 036330_2009036511.pdf.

[2] H. Minniear, J. McNabb and R. Baker, Unmanned aerial vehicle automation and control systems: Potential applications for industrial use, in: Association of Technology, Management, and Applied Engineering 2010 Conference Proceeding, pp. 82-91, available online at: http://atmae.org/conven/ATMAE2010ConfProc.pdf.

[3] P. Leggiere, First response robots: Ready for prime time, Homeland Security Magazine 7 (12) (2010) 10-11.

[4] L. A. Young, Future roles for autonomous vertical lift in disaster relief and emergency response, in: AHS International Specialists' Meeting on Advanced Rotorcraft Technology and Life Saving Activity, Aichi, Japan, Nov. 15-17, 2006.

[5] CSIP, CSIP launches mini surveillance robot into UK market, Homeland Security Magazine 6 (12) (2009) 48.

[6] GAO (Government Accountability Office), Homeland security: Observations on DHS and FEMA efforts to prepare for and respond to major and catastrophic disasters and address related recommendations and legislation, U.S. Government Accountability Office, Washington, DC, 2007.

[7] R. Baker, J. McNabb and H. Minniear, Collegiate development of crisis leaders: A strategic step toward establishing disaster preparedness/emergency response capabilities for Homeland Security, in: Proceeding Paper of Association of Technology, Management, and Applied Engineering 2010 Conference, pp. 169-180, http://atmae.org/conven/ATMAE2010ConfProc.pdf.

[8] D. G. Kamien, The McGraw-Hill Homeland Security Handbook, McGraw-Hill, NY, New York, 2006.

[9] J. Wasiluk, CBP unmanned aircraft system providing imagery above Mississippi, available online at: http://www.cbp.gov/xp/cgov/newsroom/news_releases/na tional/06152011_3.xml.

[10] H. Holub, CBP unmanned aircraft provide firefighters aerial reconnaissance, available online at: http://tucsoncitizen.com/view-from-baja-arizona/2011/06/ 17/cbp-unmanned-aircraft-provide-firefighters-aerial-reco nnaissance/.

[11] M. McCarter, Sunset for SBInet, Homeland Security Magazine 8 (2) (2011) 30-36.

[12] D. Ratner and M. A. Ratner, Nanotechnology and Homeland Security: New Weapons for New Wars, Upper Saddle River, Prentice-Hall, Inc., NJ, 2004.

[13] P. Finnegan, UAV homeland security market continues to hold potential, Homeland Security Magazine 7 (5) (2010) 19. 